\title{
O VALOR DA TOMOGRAFIA COMPUTADORIZADA NA MUDANÇA DO PARADIGMA CIRÚRGICO DO CARCINOMA EPIDERMÓIDE DE BOCA E OROFARINGE
}

\author{
THE VALUE OF COMPUTED TOMOGRAPHY ON SURGICAL PARADIGM CHANGING OF \\ SQUAMOUS CELL CARCINOMA OF MOUTH AND OROPHARYNX
}

\author{
Joel Pinheiro de Brito Junior ${ }^{1}$; Ricardo Pires de Souza²; Abrão Rapoport-ECBC-SP²; \\ Carlos Neutzling Lehn²; Ricardo Salinas Perez ${ }^{3}$; Helen Mara Rodrigues ${ }^{3}$
}

\begin{abstract}
RESUMO: Objetivo: Determinar a importância da tomografia computadorizada na mudança do paradigma cirúrgico no carcinoma espinocelular (CEC) da boca e orofaringe. Método: De 1991 e 2004, nos Departamentos de Cirurgia de Cabeça e Pescoço/ORL e Radiologia do Hospital Heliópolis, foram analisados, retrospectivamente, tomografias computadorizadas de 30 pacientes, 25 homens e cinco mulheres, com idades variando de 25 a 77 anos, todos com diagnóstico de carcinoma epidermóide do soalho da boca e/ou da língua oral e orofaringe. Todos os exames foram avaliados por dois radiologistas, separadamente, sem o conhecimento prévio do estadiamento clínico, sendo analisada a disseminação local-regional para os diferentes sítios. Para análise interobservadores, o índice Kappa foi calculado para estimar a concordância entre os mesmos, sendo o nível de significância adotado o valor de 0,05 . Resultado: A análise da concordância entre os observadores foi considerada excelente (Kappa: 0,81 - 1,00) para três subsítios (linha média, espaço mastigador e cadeia linfonodal jugulo-carotídea alta); boa (Kappa: 0,61 - 0,80) para 10 subsítios (soalho da boca e língua oral, ramo da mandíbula, trígono retromolar, espaço submandibular, loja amigdalina e cadeias linfonodais submentoniana, submandibular, jugulo-carotídea média, jugulo-carotídea baixa e espinal); moderada (Kappa: 0,41 - 0,60) para dois subsítios (corpo da mandíbula e base da língua) e não foi possível calcular o Kappa para um subsítio (cadeia linfonodal retrofaríngea). Conclusão: A concordância interobservadores predominou de boa a excelente, sendo os valores estatisticamente significantes (Rev. Col. Bras. Cir. 2007; 34(5): 290-296).
\end{abstract}

Descritores: Carcinoma de células escamosas; Neoplasias bucais; Orofaringe; Tomografia.

\section{INTRODUÇÃO}

A avaliação inicial do carcinoma espinocelular (CEC) da boca e orofaringe é realizada através da história clínica e do exame físico do paciente. O diagnóstico é confirmado pelo resultado da biopsia incisional da lesão. Os métodos de imagem complementam o exame clínico no estadiamento TNM das lesões, principalmente no que se refere à sua extensão submucosa, disseminação linfonodal e à distância.

A tomografia computadorizada permite a avaliação: de áreas, como o soalho da boca, do comprometimento da linha média, de invasão óssea mais precocemente e dos linfonodos cervicais, especialmente os retrofaríngeos, que são inacessíveis ao exame físico. Dessa forma, o diagnóstico por imagem contribui na conduta dos pacientes com carcinoma da boca e orofaringe, para o real estadiamento locoregional, imperativo no planejamento terapêutico e no prognóstico ${ }^{1,2}$. A determinação do volume do tumor é de difícil avaliação clínica, abrindo espaço para a os métodos de imagem multiplanares, como a tomografia computadorizada e a ressonância magnética ${ }^{3}$. O mais importante fator prognóstico no câncer de cabeça e pescoço é o estádio dos linfonodos cervicais, daí a fundamental importância da sua correta avaliação $0^{4}$.

Diante do exposto, torna-se evidente que o correto estadiamento TNM do CEC da boca e orofaringe por métodos de imagem seccionais é de suma importância. Porém, é possível que as diferenças no que diz respeito à formação, capacidade e experiência dos radiologistas possam interferir na precisão desses métodos. Sendo assim, a avaliação da concordância interobservadores é de grande utilidade para que se possa estimar a confiabilidade e a reprodutibilidade dos resultados desses exames, uma vez que não existe na literatura avaliação tomográfica que contribua para o estadiamento da boca e orofaringe.

\section{MÉTODO}

Foram analisados trinta (30) pacientes do Departamento de Cirurgia de Cabeça e Pescoço/Otorrinolaringologia e Radiologia do Hospital Heliópolis, Hosphel, São Paulo, dos quais 25 eram do sexo masculino, com idades variando entre 25 e 76 anos (média de 50,96 anos) e cinco eram do feminino, com idades variando entre 49 e 77 anos (média de 57,4 anos).

1. Mestre em Ciências da Saúde, Hospital Heliópolis, Hosphel, São Paulo.

2. Docente Permanente do Curso de Pós-Graduação em Ciências da Saúde, Hosphel, São Paulo.

3. Mestrando do Curso de Pós-Graduação em Ciências da Saúde, Hosphel, São Paulo.

Recebido em 05/02/2007

Aceito para publicação em 04/04/2007

Conflito interesses: nenhum

Fonte de financiamento: nenhuma

Trabalho realizado nos Departamentos de Cirurgia de Cabeça e Pescoço/Otorrinolaringologia e Radiologia do Hospital Heliópolis, Hosphel, São Paulo. 
Dos trinta pacientes, 29 eram tabagistas, 24 eram etilistas, 24 eram tabagistas e etilistas e apenas um não era tabagista nem etilista. Todos os casos eram CEC, confirmados por exame anátomo-patológico, sendo 11 casos $(36,66 \%)$ bem diferenciados, 14 casos $(46,66 \%)$ moderadamente diferenciados, quatro casos $(13,33 \%)$ pouco diferenciados, e um caso $(3,33$ $\%)$ indiferenciado, sendo todos classificados como estádio III e IV do TNM.

Dos 30 pacientes estudados, 06 foram encaminhados para tratamento cirúrgico, três para tratamento radioterápico e apenas um para tratamento quimioterápico. Em 16 casos foram associadas operação e radioterapia, em dois casos foram associadas radioterapia e quimioterapia e em dois casos operação, radioterapia e quimioterapia.

Foram considerados como critérios de inclusão a existência de CEC da boca e orofaringe, a disponibilidade de exame de imagem de tomografia computadorizada pré-tratamento para interpretação e a confirmação diagnóstica por exame anatomopatológico.

Os exames de tomografia computadorizada foram realizados em equipamentos de terceira geração. Foram utilizados dois aparelhos diferentes, porém com resolução equivalente, sendo um TCT - 500S, Toshiba Medical Incorporation, Tokyo, Japão e um CT - MAX, General Electric Medical Systems, Milwaukee, Winsconsin, Estados Unidos da América. Foram adquiridas imagens com espessura e incremento de 5,0 mm no plano axial e no plano coronal. As imagens no plano axial foram obtidas com o paciente em decúbito dorsal, adquirindo-se as imagens desde a região supra-selar, com extensão inferior até o nível da porção superior da articulação esternoclavicular, com angulação do "gantry" perpendicular à coluna aerodigestiva. Desse modo, todo o pescoço foi estudado no plano axial.

As imagens no plano coronal foram obtidas com o paciente em decúbito ventral, com extensão máxima da região cervical e com angulação máxima do "gantry" de modo crânio-caudal. As imagens foram adquiridas desde a região posterior às células mastóideas, com extensão anterior, até a porção distal dos seios frontais. Os cortes obtidos foram fotografados com janela de partes moles e com janela óssea.

Em todos os pacientes foi injetado, por via endovenosa, material de contraste iodado iônico, na dose de 1,0 a 2,0 ml/kg, com concentração de $60 \%$ e $76 \%$.

Os exames foram avaliados por dois radiologistas especializados em cabeça e pescoço, sendo denominados observador 1 e observador 2 , o primeiro com cerca de 15 anos de experiência na interpretação de exames de cabeça e pescoço e o segundo com experiência de cerca de cinco anos, ambos com mestrado e doutorado na área. A avaliação dos exames foi feita individualmente, sem o conhecimento prévio do estadiamento clínico, levando-se em consideração critérios relacionados com a extensão da lesão, com o sítio primário e com a disseminação loco-regional.

Os seguintes subsítios foram analisados:

1. Andar inferior da boca: língua, soalho, linha média, mandíbula, trígono retromolar.

2. Espaços mastigadores e mandibulares.

3. Orofaringe: loja amigdalina e base da língua.
4. Linfonodos submento-mandibulares, retrofaríngeos, júgulo-carotídeo espinal.

Para a avaliação da concordância interobservadores, na análise dos 16 subsítios estudados, foi utilizado o índice kappa (k), com os critérios de concordância mostrados na Tabela 1, assumindo-se como nível de significância o valor de 0,05 e intervalo de confiança de $95 \%$. O programa estatístico utilizado foi o EPIDAT - Análise Epidemiológica de Dados Tabulados, versão 1.0, 1994, Junta de Galícia - Organização Panamericana de Saúde.

Nos subsítios medianos/ímpares (soalho da boca e língua oral, linha média, base de língua e a cadeia linfonodal submentoniana) foi computado "n" igual ao número de casos do estudo.

Nos subsítios pares bilaterais (corpo da mandíbula, ramo da mandíbula, trígono retromolar, espaço mastigador, espaço submandibular, loja amigdalina e as cadeias linfonodais júgulo-carotídeas alta, média e baixa, submandibular, espinal e retrofaríngea) foi computado "n" duplicado considerando os dois lados analisados (direito e esquerdo) como espécimes distintos.

Conforme dito anteriormente os cortes terminavam ao nível do osso hióide em um paciente e logo abaixo em dois pacientes , impossibilitando a avaliação da cadeia júgulocarotídea média em um caso e da cadeia júgulo-carotídea média em um caso e da cadeia júgulo-carotídea baixa em três pacientes. Sendo assim, o "n" para a cadeia média foi de 58 casos e para a cadeia júgulo-carotídea baixa foi de 54 casos.

Este trabalho foi aprovado no Comitê de Ética na Pesquisa do Hospital Heliópolis, sob o nº 450 em 15/02/2006.

\section{RESULTADOS}

Quanto aos resultados, o andar inferior da boca (língua e soalho bucal, linha média e corpo da mandíbula) foram aferidos, em conjunto (Tabela 1), considerada a drenagem oncológica específica para as regiões submento-mandibular e jugulo carotídeo alta. Para as regiões laterais (Tabela 2), observou-se comportamento diverso com comprometimento júgulo carotídeo alto e espinal. Para as neoplasias da orofaringe (base de língua e amigdala) e espaço submandibular, consideramos (Tabela 3) o comportamento com disseminação uni ou bilateral das neoplasias malignas. Com relação aos linfonodos retrofaríngeos e submento-mandibular (Tabela 4), a sua aferição demonstrou a semelhança com aquela feita para as neoplasias do andar inferior da boca. Finalmente, a análise do comprometimento dos linfonodos cervicais (Tabela 5) demonstrou ser habitual a disseminação cruzada para estas neoplasias malignas.

\section{DISCUSSÃO}

Muitas lesões superficiais são detectadas por visibilização direta e não podem ser detectadas por exames de imagem $^{2,5}$, o que pode explicar o fato de ter havido discordância na avaliação de dois dos 30 casos analisados nesse estudo quanto à presença de lesão no soalho da boca e na língua oral, onde obtivemos uma concordância considerada boa, com 
Tabela 1 - Concordância interobservadores para soalho de bocallíngua oral, linha média e corpo da mandíbula.

\begin{tabular}{|c|c|c|c|c|c|}
\hline \multicolumn{6}{|c|}{ SOALHO DE BOCA/LÍNGUA ORAL $(\mathbf{N}=30)$} \\
\hline OBSERVADOR 1 & $\begin{array}{l}\text { SIM } \\
\text { NÃO } \\
\text { TOTAL }\end{array}$ & $\begin{array}{r}\text { OBS } \\
\text { SIM } \\
24 \\
1 \\
25\end{array}$ & $\begin{array}{r}\mathrm{NÃO} \\
1 \\
4 \\
5\end{array}$ & $\begin{array}{r}\text { TOTAL } \\
25 \\
5 \\
30\end{array}$ & $\begin{array}{l}\mathrm{K}=0,76 \\
\mathrm{IC} 0,402-1,0 \\
\mathrm{p}<0,001 \\
\text { Conc boa }\end{array}$ \\
\hline \multicolumn{6}{|c|}{ LINHA MÉDIA (N=30) } \\
\hline OBSERVADOR 1 & $\begin{array}{l}\text { SIM } \\
\text { NÃO } \\
\text { TOTAL }\end{array}$ & $\begin{array}{r}\text { OBS } \\
\text { SIM } \\
17 \\
0 \\
17\end{array}$ & $\begin{array}{r}\mathrm{NÃO} \\
2 \\
11 \\
13\end{array}$ & $\begin{array}{r}\text { TOTAL } \\
19 \\
11 \\
30\end{array}$ & $\begin{array}{l}\mathrm{K}=0,86 \\
\mathrm{IC} 0,507-1,0 \\
\mathrm{p}<0,001 \\
\text { Conc excelente }\end{array}$ \\
\hline \multicolumn{6}{|c|}{ CORPODA MANDÍBULA $(\mathrm{N}=60)$} \\
\hline OBSERVADOR 1 & $\begin{array}{l}\text { SIM } \\
\text { NÃO } \\
\text { TOTAL }\end{array}$ & $\begin{array}{r}\text { OBS } \\
\text { SIM } \\
4 \\
1 \\
5\end{array}$ & $\begin{array}{r}\mathrm{NÃO} \\
5 \\
50 \\
55\end{array}$ & $\begin{array}{r}\text { TOTAL } \\
9 \\
51 \\
60\end{array}$ & $\begin{array}{l}\mathrm{K}=0,52 \\
\mathrm{IC} 0,28-0,76 \\
\mathrm{p}<0,001 \\
\text { Conc moderada }\end{array}$ \\
\hline
\end{tabular}

IC = intervalo de confiança .

Conc $=$ concordância .

Tabela 2 - Concordância interobservadores para o ramo da mandíbula, trígono retromolar e espaço mastigador.

RAMODA MANDÍBULA (N=60)

\begin{tabular}{llrrrl} 
& & OBSERVADOR-2 & & \\
OBSERVADOR 1 & SIM & NÃO & TOTAL & $\mathrm{K}=0,66$ \\
& SIM & 1 & 0 & 1 & IC $0,421-0,897$ \\
& NÃO & 1 & 58 & 59 & $\mathrm{p}<0,001$ \\
& TOTAL & 2 & 58 & 60 & Conc boa \\
\hline
\end{tabular}

TRÍGONO RETROMOLAR $(\mathbf{N}=60)$

\begin{tabular}{|c|c|c|c|c|c|}
\hline \multicolumn{6}{|c|}{ OBSERVADOR - 2} \\
\hline \multirow{4}{*}{ OBSERVADOR 1} & & SIM & NÃO & TOTAL & $K=0,66$ \\
\hline & SIM & 1 & 1 & 2 & IC $0,421-0,897$ \\
\hline & NÃO & 1 & 58 & 58 & $\mathrm{p}<0,001$ \\
\hline & TOTAL & 1 & 59 & 60 & Conc boa \\
\hline
\end{tabular}

ESPAÇO MASTIGADOR $(\mathbf{N}=60)$

\begin{tabular}{llrrrl} 
& & OBSERVADOR-2 & & \\
OBSERVADOR 1 & SIM & NÃO & TOTAL & $\mathrm{K}=1,00$ \\
& SIM & 1 & 0 & 1 & IC $0,747-1,0$ \\
& NÃO & 0 & 59 & 59 & $\mathrm{p}<0,001$ \\
& TOTAL & 1 & 59 & 60 & Conc excelente \\
\hline
\end{tabular}

$\mathbf{I C}=$ intervalo de confiança.

Conc $=$ concordância .

kappa de 0,76 , coincidentes com outras séries que apresentam os mesmos valores ${ }^{6-8}$.

O kappa é uma medida de concordância interobservadores que avalia o grau de concordância além do que seria esperado tão somente ao acaso. Essa medida pode variar de $-1 \mathrm{a}+1$, sendo que o valor máximo $(+1)$, representa concordância perfeita; o valor zero, indica que a concordância foi exatamente a esperada pelo acaso. Um eventual valor menor que zero (negativo), sugere que a concordância foi menor do que aquela esperada pelo acaso e, portanto, sugere discordância, porém seu valor não tem interpretação como intensidade de discordância. Nesse trabalho o p foi estatisti- 
Tabela 3 - Concordância interobservadores para o espaço submandibular, loja amigdalina e base da língua.

\section{ESPAÇOSUBMANDIBULAR $(\mathbf{N}=60)$}

OBSERVADOR - 2

$\begin{array}{llrrrr} & & \text { SIM } & \text { NÃO } & \text { TOTAL } & \text { K=0,65 } \\ \text { OBSERVADOR 1 } & \text { SIM } & 11 & 2 & 13 & \text { IC } 0,397-0,896 \\ & \text { NÃO } & 6 & 41 & 47 & \text { p }<0,001 \\ & \text { TOTAL } & 17 & 43 & 60 & \text { Conc boa }\end{array}$

\begin{tabular}{|c|c|c|c|c|c|}
\hline \multicolumn{6}{|c|}{ LOJAAMIGDALINA $(\mathrm{N}=60)$} \\
\hline \multirow{5}{*}{ OBSERVADOR 1} & & \multicolumn{4}{|c|}{ OBSERVADOR - 2} \\
\hline & & SIM & NÃO & TOTAL & $\mathrm{K}=0,64$ \\
\hline & SIM & 3 & 1 & 4 & IC 0,389-0,891 \\
\hline & NÃO & 2 & 54 & 56 & $\mathrm{p}<0,001$ \\
\hline & TOTAL & 5 & 55 & 60 & Conc boa \\
\hline \multicolumn{6}{|c|}{ BASE DA LÍNGUA $(\mathbf{N}=30)$} \\
\hline \multirow{5}{*}{ OBSERVADOR 1} & & OBS & & & \\
\hline & & SIM & NÃO & TOTAL & $\mathrm{K}=0,55$ \\
\hline & SIM & 7 & 4 & 11 & IC $0,198-0,906$ \\
\hline & NÃO & 2 & 17 & 19 & $p=0,002$ \\
\hline & TOTAL & 9 & 21 & 30 & Conc moderada \\
\hline
\end{tabular}

IC = intervalo de confiança.

Conc $=$ concordância

Tabela 4 - Concordância interobservadores para cadeias linfonodais retrofaríngea, submentoniana e submandibular.

\begin{tabular}{|c|c|c|c|c|c|}
\hline \multicolumn{6}{|c|}{ CADEIALINFONODAL RETROFARÍNGEA(N=60) } \\
\hline \multirow{5}{*}{ OBSERVADOR 1} & & OBS & & & \multirow{5}{*}{$\begin{array}{l}\text { Não foi possível } \\
\text { calcular o Kappa }\end{array}$} \\
\hline & & SIM & NÃO & TOTAL & \\
\hline & SIM & 0 & 0 & 0 & \\
\hline & NÃO & 0 & 60 & 60 & \\
\hline & TOTAL & 0 & 60 & 60 & \\
\hline \multicolumn{6}{|c|}{ CADEIA LINFONODAL SUBMENTONIANA $(\mathrm{N}=30)$} \\
\hline \multirow{5}{*}{ OBSERVADOR 1} & & OBS & & & \multirow{5}{*}{$\begin{array}{l}\mathrm{K}=0,71 \\
\mathrm{IC} 0,371-1,0 \\
\mathrm{p}<0,001 \\
\text { Conc boa }\end{array}$} \\
\hline & & SIM & NÃO & TOTAL & \\
\hline & SIM & 3 & 0 & 3 & \\
\hline & NÃO & 2 & 25 & 27 & \\
\hline & TOTAL & 5 & 25 & 30 & \\
\hline \multicolumn{6}{|c|}{ CADEIALINFONODALSUBMANDIBULAR $(\mathbf{N}=\mathbf{3 0})$} \\
\hline \multirow{5}{*}{ OBSERVADOR 1} & & OBSI & & & \multirow{5}{*}{$\begin{array}{l}\mathrm{K}=0,71 \\
\text { IC } 0,1459-0,955 \\
\mathrm{p}<0,001 \\
\text { Conc boa }\end{array}$} \\
\hline & & SIM & NÃO & TOTAL & \\
\hline & SIM & 10 & 1 & 11 & \\
\hline & NÃO & 5 & 44 & 49 & \\
\hline & TOTAL & 15 & 45 & 60 & \\
\hline
\end{tabular}

IC = intervalo de confiança .

Conc $=$ concordância .

camente significante em todos os subsítios avaliados, mesmo quando a concordância foi mais baixa; para valores de kappa menores ou igual a zero os valores de p podem ser maiores que 0,05 , não tendo portanto significância estatística ${ }^{9}$.

Para a variável linha média, obtivemos uma concordância excelente $(\mathrm{kappa}=0,86)$, o que nos permite infe- rir que a tomografia computadorizada é um ótimo método para detectar o seu envolvimento pelo tumor, fato que na prática clínica tem uma importância fundamental já que interfere diretamente na conduta cirúrgica (glossectomia total ou parcial $)^{1}$ e conseqüentemente na qualidade de vida do paciente. 
Tabela 5 - Concordância interobservadores para a cadeia linfonodal jugulo-carotídea alta, média e baixa e cadeia linfonodal espinal.

\begin{tabular}{|c|c|c|c|c|c|}
\hline \multicolumn{4}{|c|}{ CADEIALINFONODALJUGULO-CAROTÍDEAALTA(N=60) } & \multirow[b]{3}{*}{ TOTAL } & \\
\hline \multirow{5}{*}{ OBSERVADOR 1} & & OBSI & & & \\
\hline & & SIM & NÃO & & $\mathrm{K}=0,81$ \\
\hline & SIM & 17 & 3 & 20 & IC $0,459-1,0$ \\
\hline & NÃO & 2 & 38 & 40 & $p<0,001$ \\
\hline & TOTAL & 19 & 41 & 60 & Conc excelente \\
\hline
\end{tabular}

\begin{tabular}{|c|c|c|c|c|c|}
\hline \multicolumn{4}{|c|}{ CADEIA LINFONODALJUGULO-CAROTÍDEA MÉDIA(N=58) } & \multirow[b]{3}{*}{ TOTAL } & \multirow[b]{3}{*}{$\mathrm{K}=0,80$} \\
\hline \multirow{5}{*}{ OBSERVADOR 1} & & OBSE & & & \\
\hline & & SIM & NÃO & & \\
\hline & SIM & 11 & 2 & 13 & IC $0,544-1,0$ \\
\hline & NÃO & 2 & 43 & 45 & $\mathrm{p}<0,001$ \\
\hline & TOTAL & 13 & 45 & 58 & Conc boa \\
\hline
\end{tabular}

CADEIA LINFONODAL JUGULO-CAROTÍDEA BAIXA $(\mathbf{N}=54)$

\begin{tabular}{|c|c|c|c|c|c|}
\hline & & OBS & & & \\
\hline & & SIM & NÃO & TOTAL & $K=0,64$ \\
\hline OBSERVADOR 1 & SIM & 3 & 3 & 6 & IC $0,391-0,889$ \\
\hline & NÃO & 2 & 17 & 19 & $\mathrm{p}<0,001$ \\
\hline & TOTAL & 9 & 21 & 30 & Conc boa \\
\hline CADEIA LINFON & L ESPIN & $\mathrm{N}=60$ & & & \\
\hline & & OBS & & & \\
\hline & & SIM & NÃO & TOTAL & $\mathrm{K}=0,78$ \\
\hline OBSERVADOR 1 & SIM & 4 & 2 & 6 & IC $0,536-1,0$ \\
\hline & NÃO & 0 & 54 & 54 & $\mathrm{p}<0,001$ \\
\hline & TOTAL & 4 & 56 & 60 & Conc boa \\
\hline
\end{tabular}

$\mathbf{I C}=$ intervalo de confiança .

Conc $=$ concordância .

Para as variáveis corpo e ramo da mandíbula, a concordância obtida nesse estudo foi moderada (kappa=0,52) e boa (kappa $=0,66$ ), discordantes de série que revela resultado desprezível (Kappa=0,04) para o corpo da mandíbula e boa (kappa $=0,66)$ para o ramo ${ }^{9}$ e encontrou uma concordância moderada para ambas as variáreis (kappa $=0,49$ e 0,47, respectivamente $)^{6}$. Na avaliação da base da língua, obtivemos uma concordância moderada (kappa $=0,55$ ), discordante da literatura (kappa $=0,64)^{6}$.

$\mathrm{Na}$ avaliação das cadeias linfonodais obtivemos uma concordância excelente para a cadeia júgulo-carotídea alta e boa para as demais, resultados semelhantes à literatura ${ }^{7,8,10,11}$, com predomínio de concordâncias boas e excelentes, reafirmando a validade do método no estadiamento cervical e sua utilidade na prática clínica, já que o envolvimento linfonodal interfere na conduta cirúrgica e no prognóstico do pacien$\mathrm{te}^{4,11}$.

Em resumo, na série estudada, a concordância entre os observadores variou de moderada a excelente, com predomínio de boa e excelente. Dos 16 sítios analisados, a concordância foi considerada excelente em três subsítios (linha média, espaço mastigador e cadeia linfonodal jugulo-carotídea alta), boa em 10 subsítios (soalho da boca e língua oral, ramo da mandíbula, trígono retromolar, espaço submandibular, loja amigdalina, cadeias linfonodais submentoniana, submandibular, jugulo-carotídea média, jugulo-carotídea baixa e espinal), moderada em dois subsítios (corpo da mandíbula e base de língua) e não foi possível calcular o kappa para um subsítio (cadeia linfonodal retrofaríngea). Em todos esses subsítios o $p$ foi menor que 0,05 , sendo, portanto, estatisticamente significante.

É importante salientar que para a realização da análise interobservadores é necessário que os observadores tenham vivência e treinamento específico na área, como ocorreu no presente estudo. Do contrário, poderia ocorrer um viés na avaliação, levando a resultados imprecisos, não pela falta de sensibilidade do método (tomografia computadorizada), mas pela falta de experiência dos observadores.

Em dois subsítios avaliados (corpo da mandíbula e base da língua) a força de concordância foi moderada (kappa igual a 0,52 e 0,55 , respectivamente) e o valor de p foi estatisticamente significante $(\mathrm{p}<0,05)$, podendo-se inferir, a partir desses resultados, que a tomografia não foi tão eficaz na avaliação desses sítios quanto dos demais. Em relação à cadeia linfonodal retrofaríngea, não foi possível calcular o kappa, mas os dois observadores concordaram que não houve comprometimento desse subsítio em todos os casos. Situação idêntica foi referida por outro autor em relação à cadeia linfonodal 
retrofaríngea, o que pode ser justificado pelo fato do teste Kappa ser eficaz na avaliação da presença de um sinal específico; nas variáveis em que o sinal avaliado não está presente, em todos os casos ou na maioria deles, pode acontecer que o valor do Kappa seja negativo ou mesmo não possa ser calculado.

Como resultado desse trabalho obtivemos um grau de concordância interobservadores considerado satisfatório (boa e excelente) em 13 subsítios dos 16 analisados. Em um subsítio não foi possível calcular o kappa, não sendo possível uma análise satisfatória com o uso deste coeficiente e em dois subsítios a concordância foi moderada. Portanto, diante de uma concordância satisfatória em 13 dos 15 subsítios analisáveis pelo método, podemos inferir que a tomografia computadorizada mostrou-se um método eficaz para a avaliação do estadiamento loco-regional do carcinoma epidermóide do soalho da boca e da língua oral. Entretanto, obtivemos uma concordância considerada insuficiente para o corpo da man- díbula e a base da língua e como a correta avaliação dessas estruturas é fundamental para o planejamento cirúrgico, em um futuro trabalho propõe-se a realização de um estudo correlacionando a tomografia computadorizada com a ressonância magnética e com os achados cirúrgicos e anatomopatológicos.

A concordância interobservadores em relação ao estadiamento loco-regional do carcinoma epidermóide da boca e orofaringe por meio da análise da tomografia computadorizada, em uma escala que varia de desprezível a excelente, para os subsítios avaliados foi:

- Boa (língua oral, soalho bucal, ramo da mandíbula, trígono retromolar, espaço submandibular, loja amigdalina, linfonodos submentonianos, submandibular, júgulo carotídeo médio, júgulo carotídeo baixo e espinal).

- Excelente: linha média, espaço mastigador, linfonodo jugulo-carotídeo.

- Moderada: Corpo da mandíbula, base da língua.

\begin{abstract}
Background: The aim of this study is to evaluate the interobserver agreement, using computed tomography, regarding the local and regional extension of mouth and oropharynx squamous cell carcinoma. Method: From 1991 through 2004, thirty patients, with available CT examinations, were examined at the Head and Neck Surgery, Otorhinolaryngology and Imaging Departments of Heliópolis Hospital, São Paulo, Brazil. They were included in this retrospective non-randomized study. We performed biopsies and histopathologic examinations in all cases. There were 25 males and 5 females, with ages ranging from 25 to 77 years. Two radiologists evaluated all cases individually, without previous clinical staging data, analyzing local and regional sites of lesion dissemination. No patients had received any previous treatment up to the moment of CT examination. Results: We calculated the kappa index by assessing agreement between the two observers, and the significance level was assumed 0.05. Agreement was excellent (Kappa: 0.81-1.00) in three analyzed subsets (middle line, masticator space and high jugular lymph node chains). It was good (Kappa: $0.61-0.80$ ) in ten subsets (floor of the mouth and oral tongue, mandibular branches, retromolar trigone, submandibular space, tonsillar fossa and submentonian, submandibular, middle jugular, low jugular and spinal lymph node chains). It was moderate (Kappa: 0.41 - 0.60) in two subsets (mandibular body and base of the tongue), and in one subset the analysis was not possible using this coefficient where the Kappa index was not feasible. Conclusion: The interobserver agreement was of significant statistical value, predominating from good to excellent.
\end{abstract}

Key words: Carcinoma, squamous cell; Mouth neoplasms; Oropharynx; Tomography.

\section{REFERÊNCIAS}

1. Larsson SG, Mancuso A, Hanafee W. Computed tomography of the tongue and floor of the mouth. Radiology. 1982;143(2):493500 .

2. Muraki AS, Mancuso AA, Harnsberger HR, Johnson LP, Meads GB. CT of the oropharynx, tongue base and floor of the mouth: normal anatomy and range of variations, and applications in staging carcinoma. Radiology. 1983;148(3):725-31.

3. Hermans R, Feron M, Bellon E, Dupont P, van den Boagaert W, Baert AL. Laryngeal tumor volume measurements determined with CT: a study on intra- and interobserver variability. Int J Radiat Oncol Biol Phys. 1998;40(3):553-7.

4. Shah JP, Kowalski LP. Cirurgia de cabeça e pescoço. $2^{\mathrm{a}}$ ed. Rio de Janeiro: Revinter; 2000.

5. Som PM, Curtin HD. Head and neck imaging. $4^{\text {th }}$ ed. St. Louis: Mosby; 2003.

6. Yamashiro I. Avaliação da concordância interobservadores na análise do carcinoma epidermóide da loja tonsilar por meio da tomografia computadorizada [dissertação]. São Paulo (SP): Curso de Pós-Graduação em Ciências da Saúde do Complexo Hospitalar Heliópolis; 2006.

7. Paes Jr AJO. Avaliação da concordância interobservadores no estadiamento do carcinoma da base da língua por meio de tomografia computadorizada [dissertação]. São Paulo (SP): Curso de Pós-Graduação em Ciências da Saúde do Complexo Hospitalar Heliópolis; 2004.

8. .Gonzales FM. Avaliação da concordância interobservadores no estadiamento do carcinoma do trígono retromolar por meio de tomografia computadorizada [disasertação]. São Paulo(SP): Curso de Pós-Graduação em Ciências da Saúde do Complexo Hospitalar Heliópolis; 2005.

9. . Jekel JF, Elmore JG, Katz DL. Epidemiologia, estatística e medicina preventiva. $2^{\mathrm{a}}$ ed. Porto Alegre: Artmed; 2005.

10. Paes Jr AJO. Análise da concordância interobservadores na avaliação da extensão linfonodal do carcinoma das vias aerodigestivas superiores por meio da tomografia computadorizada [dissertação]. São Paulo (SP): Universidade de São Paulo; 2006. 
11. Kurokawa H, Yamashita Y, Takeda S, Zhang M, Fukuyama H, Takahashi T. Risk factors for late cervical lymph node metastases in patients with stage I or II carcinoma of the tongue. Head Neck. 2002;24(8):731-6.

Como citar este artigo:

Brito Jr JP, Souza RP, Rapoport A, Lehn CN, Perez RS, Rodrigues HM. O valor da tomografia computadorizada na mudança do paradigma cirúrgico do carcinoma epidermóide de boca e orofaringe. Rev Col Bras Cir. [periódico na Internet] 2007 Set-Out;34(5). Disponível em URL: www.scielo.br/rcbc
Endereço para correspondência:

Abrão Rapoport - Rua Iramaia, $n^{\circ} 136$

Jd. Europa

01450-020 - São Paulo - SP

E-mail: ararapoport@terra.com.br 